\title{
Electrical Behavior of Solar Cell Based on ZnO/PS
}

\author{
${ }^{1}$ Z.Yamlahi Alami, ${ }^{1}$.El khamkhami, ${ }^{2}$ M. Salem and ${ }^{2}$ M.Gaidi \\ ${ }^{1}$ laboratoire de la matière condensée, dept de physique, faculté de sciences Avenue de Sebta, Mhannech \\ I 93002 - Tétouan - Maroc \\ ${ }^{2}$ Laboratoire de Photovoltaïque Centre de Recherches et des Technologies de l'Energie, Technopole de \\ Borj-Cédria, BP 95, 2050 Hammam-Lif, Tunisia \\ a.yamlahizineb@gmail.com
}

\begin{abstract}
Because of high loss of photovoltaic conversion due to reflection of incident photon by the silicon surface, we proposed in this work a single heterojunction solar cell model based on porous silicon (PS) and Zinc oxide to minimize this loss, which the $\mathrm{ZnO}$ could act like front n-layer as well as antireflection coating saving processing cost and complexity. In the experiment the $\mathrm{ZnO}$ layer was deposited by spray pyrolysis method on porous silicon (PS) using nitrate precursors with different molar concentration. The proposed treatment has also demonstrated a significant decrease in the reflectivity of $\mathrm{ZnO} / \mathrm{PS}$. To simulate a solar cell based on porous silicon and zinc oxide, we have used PC1D software. The variation of internal and external quantum efficiency, and I-V characteristics have been done by changing the reflectivity of ARC layer, The I-V results obtained by PC1D simulation was compared with the experiment results: Simulations anticipated conversion efficiency was $14,5 \%$ while by experiments was $12 \%$ for the device fabricated with $0.25 \mathrm{M}$.
\end{abstract}

Keywords-component; ZnO, PC1D, Characteristic I-V.

\section{Introduction}

The studies have focused on solar cells as an alternative energy sources to decrease the fossil fuels 'uses, but the high price of $1 \mathrm{KWh}$ of electricity generated by a solar cell prevents the wide propagation of this technology so the researches have concentrated on reducing this cost by using a cheaper materials as a cover layer .

Thin film nanostructure has unique proprieties make it capable to play a role as a cover layer. Zinc oxide $(\mathrm{ZnO})$ is one of the most extensively studied materials as an alternative photo anode to TiO2 [1]. ZnO has a wide range of properties that depend on doping, including conductivity which may change at will from metallic to insulting (including n-type and p-type conductivity), high transparency [2], Piezoelectricity [3], room-temperature ferromagnetism [4], and huge magneto-optic effect [5] ,etc...

Without much effort, $\mathrm{ZnO}$ can be grown into many different nanoscale forms, thus allowing various novel devices to be achieved. It has a wide band gap energy of $3.37 \mathrm{eV}$, the larger exciton binding energy of 60 meV guarantees efficient luminescent and photovoltaic characteristics , a wide range resistivity, high electron Hall mobility (200 cm2 .V .s-1), low toxicity, natural abundance, and highly stable wurtzite structure . 
ZnO thin films have been prepared using various methods as magnetron sputtering [6], spray plasma [7], sol gel spin coating[8], pulsed laser deposition[9], metal-organic chemical vapor deposition and spray pyrolysis[2].

Among this method spray pyrolysis is a versatile technique for the deposition of metal oxides because of its low cost and easy process control which gives the possibility of obtaining films with the required properties for different applications [10].

In this paper, we propose a new model of potentially cost effective and high efficiency single heterojunction solar cells based on porous silicon (PS) (rear region) and the emerging II-VI material $\mathrm{ZnO}$ (front region). The $\mathrm{ZnO}$ grown on porous silicon can work as an active n-layer as well as antireflection coating.

This model was simulated by PC1D software, the different electrical and optical characteristics as internal, external quantum and efficiencies are investigated by varying different key parameters.

\section{Experimental}

Experiments were carried out on p-type, boron-doped multicrystalline silicon substrate with a thickness of $350 \mu \mathrm{m}$ and a resistivity of 0.5 to 2 ? $\mathrm{cm}$. Porous silicon was formed by electrochemical etching $\mathrm{mc}-\mathrm{Si}$ wafers in HF 48\% /ethanol ( $1 \mathrm{~V}: 2 \mathrm{~V}$ ) at a constant current density of $15 \mathrm{~mA} / \mathrm{cm} 2$ for an etching time of 10 min. Immediately after PS formation, the samples were rinsed with DI water and dried under N2 flux[10].

ZnO thin films were prepared by spray pyrolysis process from a solution with different molar concentrations of $\mathrm{Zn}(\mathrm{NO} 3) 2,6 \mathrm{H} 2 \mathrm{O}$ precursor $(0.05 \mathrm{M}, 0.1 \mathrm{M}, 0.15 \mathrm{M}, 0.2 \mathrm{M}$ and $0.25 \mathrm{M})$ in $25 \mathrm{ml}$ of deionized water. The spray system used in this work was fully described and schematically presented in [11]. The $\mathrm{Zn}(\mathrm{NO} 3) 2,6 \mathrm{H} 2 \mathrm{O}$ solution was sprayed with a solution spray rates of $2 \mathrm{ml} / \mathrm{min}$ onto a preheated silicon porous substrate at $500^{\circ} \mathrm{C}$ during $10 \mathrm{~min}$, using compressed air as a carrier gas. The nozzle to substrate distance was about $45 \mathrm{~cm}$. The $\mathrm{ZnO}$ thin Films thus obtained are transparent, exhibiting a good adherence to the substrate surfaces.

The reflectivity spectra of the different surface were measured using a LAMBDA 950 UV/ Vis/NIR spectrophotometer equipped with an integrating sphere. The effective minority lifetime (teff) was measured using the Sinton WCT-120 lifetime tester in quasi-steady-state photoconductance (QSSPC) mode.

\section{Simulation}

Our experiment results were introduced in the PC1D simulation software to simulate the solar cell based on porous silicon and $\mathrm{ZnO}$ using the parameters illustrate in table 1.

Table 1 resumes the parameters was optimized for the solar cell based on the some values obtained in the experiments characterization and the other based on the $\mathrm{ZnO}$ proprieties.

The several adjustable parameters in the PC1D can be iterated, to introduce the EQE, IQE, I-V and efficiency of solar cell. 


\begin{tabular}{|c|c|}
\hline Varying parameters & Value \\
\hline Device parameters & \\
\hline Device area & $1 \mathrm{~cm}^{2}$ \\
\hline Surface texturing & None \\
\hline Surface charge & None \\
\hline Front reflectance & $18 \%-9 \%$ \\
\hline Emitter contact resistance & $10^{-6} \Omega$ \\
\hline Base contact resistance & $0.4 \Omega$ \\
\hline Front region $(\mathrm{ZnO})$ & \\
\hline Region thickness & $0.015 \mu \mathrm{m}$ \\
\hline Electron/hole mobility & $50 \mathrm{~cm}^{2} / \mathrm{Vs}$ \\
\hline Dielectric constant & 8.66 \\
\hline Bandgap & $3,64-3,14 \mathrm{eV}$ \\
\hline Refractive index & $2-2,38$ \\
\hline n-type background doping & $3.10^{13}$ \\
\hline bulk electron/hole recombination time & $\begin{array}{c}1,2-1,6-5-57- \\
90 \mu \mathrm{s}\end{array}$ \\
\hline electron/hole front surface recombination velocity & $1.107 \mathrm{~cm} / \mathrm{s}$ \\
\hline Rear region $(\mathrm{Si})$ & \\
\hline Region thickness & $350 \mu \mathrm{m}$ \\
\hline Carrier mobilities & $\begin{array}{l}\text { From internal } \\
\text { model of PC1D }\end{array}$ \\
\hline Dielectric constant & 11,9 \\
\hline Bandgap & $1,124 \mathrm{eV}$ \\
\hline Intrinsic concentration at $300 \mathrm{~K}$ & $1.1010 \mathrm{~cm}^{-3}$ \\
\hline p-type background doping & $1.1016 \mathrm{~cm}^{-3}$ \\
\hline peak rear $\mathrm{p}$-type doping & $3.1018 \mathrm{~cm}^{-3}$ \\
\hline bulk electron/hole recombination time & $100 \mu \mathrm{s}$ \\
\hline electron/hole front surface recombination velocity & $1.1016 \mathrm{~cm} / \mathrm{s}$ \\
\hline electron/hole rear surface recombination velocity & $300 \mathrm{~cm} / \mathrm{s}$ \\
\hline
\end{tabular}

\section{Results}

\subsection{Experimental results}

Some parameters were changed when we have changed the molar concentration of the $\mathrm{Zn}$, these parameters have influenced on the simulated results as EQE, IQE, I-V, and solar cell's efficiency, where the reflectivity parameter of the thin film ZNO/PS is the most important. Lower is the reflection from the front surface higher is the EQE and IQE.

Figure.1 Shows AFM images of the ZnO/PS surface treatment and a reference sample (untreated PS layer) for comparison. It can be seen from the different AFM topography a clear evolution of the microstructure and the surface has an important roughness, the reported roughness (RMS) was found to be around 425 $\mathrm{nm}$ for PS wafer and increases to $556 \mathrm{~nm}$ after ZnO deposition. The RMS enhancement is attributed to the increase of the nanoparticle size when increasing $\mathrm{ZnO}$ molar concentration. 


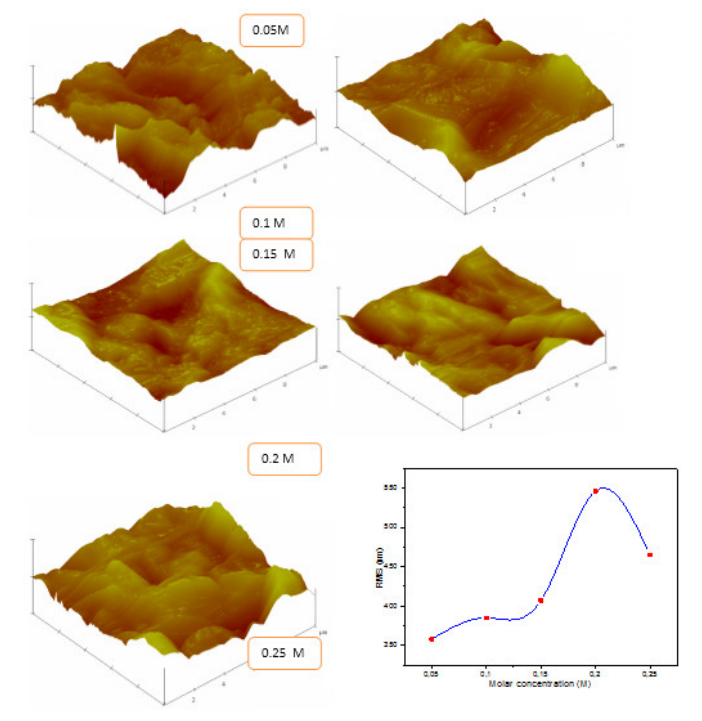

Figure.1 (3D) AFM images of PS and ZnO/PS with various $\mathrm{Zn}$ concentrations

Figure. 2 shows that the average reflectivity reduced from $18 \%$ to $9 \%$ when the molar concentration decreases from $0.25 \mathrm{M}$ to $0.05 \mathrm{M}$. The measured refractive index and extinction coefficient values changed, the former from 2 to 2.38 which is very close to the ideal value and the later from 0.01 to 0.04 for $\lambda=500$ $\mathrm{nm}$ [10]. This can eliminate requirement of additional antireflection coating, resulting in reduced fabrication process steps and cost. For Babar et al. [14] the refractive index of $\mathrm{ZnO}$ at $600 \mathrm{~nm}$ equal 2 is very suitable to play role of antireflection coating AR for $\mathrm{Si}$. The effective carrier lifetime also another important parameter influences on the simulated result (figure 3 shows the values of the each sample obtained on the experimental results).

The different experiment results obtained have introduced using PC1D to simulate the different parameters as EQE, IQE, I-V, and solar cell's efficiency.

B. Hassain in his work [14] also observes that the VOC, Jsc and efficiency ( $\eta$ ) have influence by change of ZnO thickness using PC1D software, where the optimized modeled thickness was obtained as $0.1 \mu \mathrm{m}$ for minimum reflection, they have achieved an efficiency of $19,3 \%$, a $\mathrm{Voc}$ of $622 \mathrm{mV}$, a Jsc of $38,5 \mathrm{~mA}$.

\subsection{Simulated results}

We can distinguish between two types of quantum efficiency: external and internal (EQE and IQE), in other words the external quantum efficiency is defined as the ratio of the charge carrier's number collected by the solar cell to the photon's number of a given energy incident on the solar cell from outside. The second kind (IQE) is the ratio of the charge carrier's number collected by the solar cell to the number of photons of a given energy that shine on the solar cell from outside and are absorbed by the cell. A low value of thin film's IQE indicates that the active layer of the solar cell is unable to make good use of the photons. 


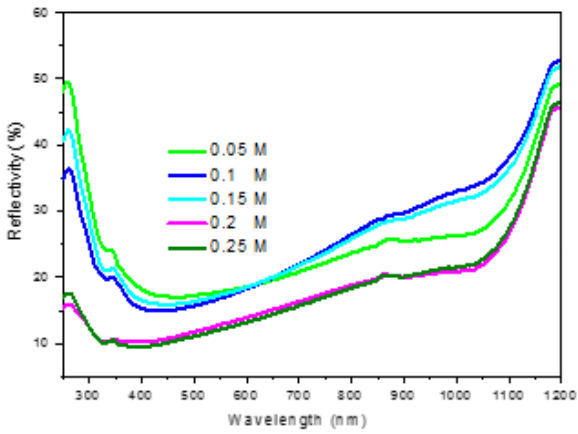

Figure.2 Total reflectivity ZnO/PS film

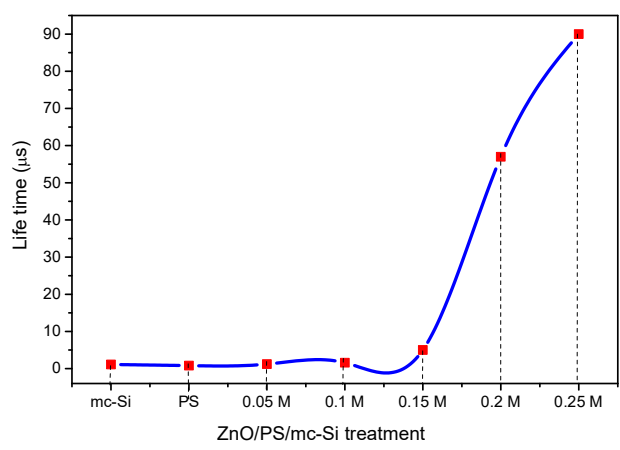

Figure 3: Effective minority carrier lifetime evolution of $\mathrm{ZnO} / \mathrm{PS}$

Figure 4 shows the variation of IQE with different front back. The graph indicates that the IQE augments with the increase in the molar concentration of $\mathrm{ZnO}$ thin film due to the low value of reflection. The low reflectivity of the thin film $\mathrm{ZnO} / \mathrm{SP}$ fabricated with a high concentration of $\mathrm{Zn}(0.25 \mathrm{M}$ and $0.2 \mathrm{M})$ also influences on the wide of the band. So the thin film with a high molar concentration has absorbed the photons with long wavelength. In a similar way EQE also increases with the increasing of molar concentration (these results are shown in Figure 5). The absorption of high energy photons in $\mathrm{ZnO}$ is mainly responsible for poor EQE at short wavelengths.

Table 2 The Eg values of different $n-Z n O$

\begin{tabular}{|c|c|c|c|c|c|}
\hline $\begin{array}{c}\text { Sample molar } \\
\text { concentration (M) }\end{array}$ & 0.05 & 0.1 & 0.15 & 0.2 & 0.25 \\
\hline $\mathrm{Eg}(\mathrm{eV})$ & 3.64 & 3.56 & 3.34 & 3.15 & 3.15 \\
\hline
\end{tabular}

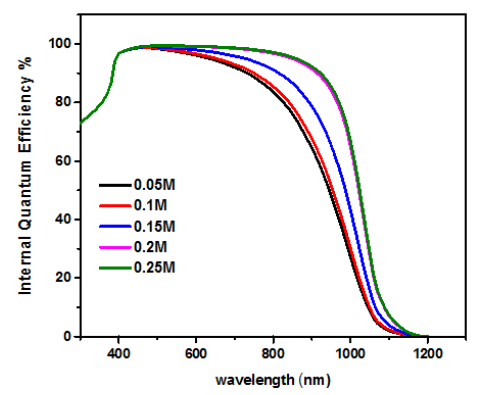

Figure. 4 Internal quantum efficiency (IQE) of the solar cell device with different front back.

The EQE was clearly improved in which the values of EQE increased from 80 to $90 \%$ at a wavelength of $500 \mathrm{~nm}$. This result might be explained by the decreased reflectivity of ZnO/SP. Babar has explained in his work [13] the improvement of EQE and front surface reflection with taking into account antireflection effect of $\mathrm{ZnO}$ layer, therefore, the EQE was increasing from $84 \%$ to $98 \%$ around wavelength of $600 \mathrm{~nm}$ when he adds the $\mathrm{ZnO}$ layer.

When the Eg decreases, the wideness of band gets larger, therefore, the IQE and EQE got influenced. All this due to fact the solar cell could absorb more the photons characterized with lower energy. Thus, the number of photons absorbed increases, (table 2 shows the value of the Eg of each sample).The decrease in band gap energy with increase in molar concentration could be due to increase in crystalline size and reduction of defect sites and increase in film thickness $[2,10]$. 
Figure 6 shows the comparison between the experimental I-V curve and the simulated one. The slight increase in the I-V simulated curve due to the front contact of solar cell is ideal in the simulation.

Based on life time values obtained in the experiment and reflectivity data, we have stimulated by PC1D the I-V spectra of ZnO /SP heterojunction under AM1.5 illumination.

The simulated I-V curves of the $\mathrm{HJ}$ solar cell of $\mathrm{ZnO} / \mathrm{SP}$ treated with different molar concentration are shown in figure 7. The enhancement in the value of the short-circuit current density (JSc) from $26,8 \mathrm{~mA}$ to $34,77 \mathrm{~mA}$ and the open circuit voltage Voc from $526,1 \mathrm{mV}$ to $536,9 \mathrm{mV}$. The Jsc increase monotonically with increased $\mathrm{Zn}$ molar concentration because of significant increase in number available photons in space charge region as shown in figure 4.

The I-V curves simulated shows the dependency between I-V behavior and different parameters obtained in the experience those parameters can be cited as follows the reflectivity, life time and band gap of $\mathrm{ZnO}$ thin film deposited on Si/porous (table 1 and table 2), optimizing these three parameters yields an optimum Jsc and Voc .

Salem et al. in his work [10] has demonstrated an improvement in the short-circuit current density (Jsc) from 23.4 to $30 \mathrm{~mA} / \mathrm{cm} 3$, and the open circuit voltage Voc got increased from $526.1 \mathrm{mV}$ to $536.9 \mathrm{mV}$, in which these values are close to the result obtained in this work.

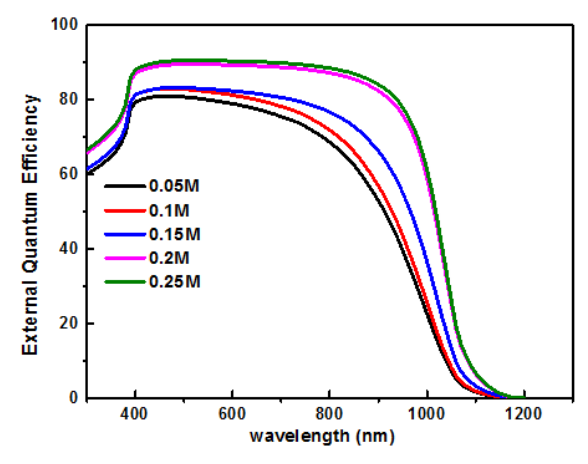

Figure.5 External quantum efficiency (EQE) of the solar cell device with different front back

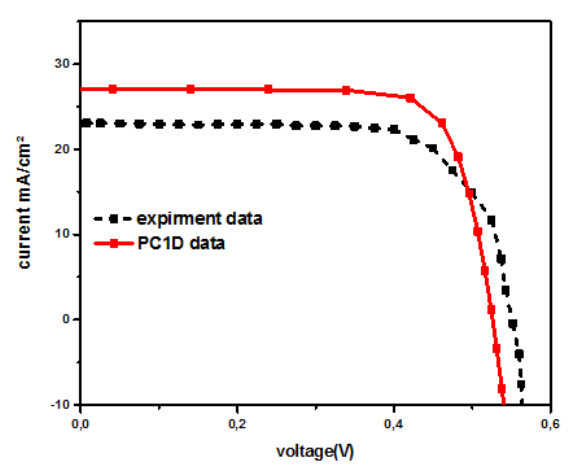

Figure 6 I-V Experiment and PC1D spectra of $\mathrm{ZnO} / \mathrm{PS}$ of $0.05 \mathrm{M}$ sample

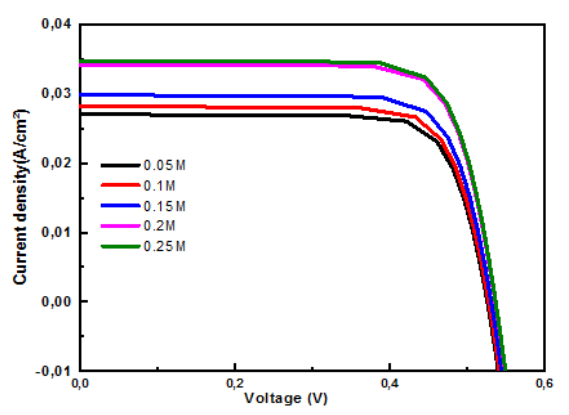

Figure .7 I-V Characteristics of ZnO / PS heterojunction under AM1.5 M 
The quality of the solar cell is defined by it efficiency $(\eta)$ of light conversion; the efficiency is the ratio of the energy output from the cell (Pmax) to the input energy from the sun (Pin). It was calculated by using the equation:

$$
\eta=\frac{\operatorname{Im}^{*} V m}{P i}
$$

Where $\mathrm{Vm}$ is the value of the voltage for the maximum power from a solar cell, and $\mathrm{Im}$ is the value of the current for the maximum power from a solar cell.

The $\eta$ values go up from $11.1 \%$ to $14.5 \%$. The proposed treatment demonstrates a significant enhancement of this parameter after $\mathrm{ZnO} / \mathrm{PS}$ treatment.

On the other hand the result of I-V and $\eta$ in the experiment results are shown in the table 3 , as it is seen there is the slight enhancement in the simulation results due to the ideal solar cell.

Table 3 Photovoltaic parameters from illuminated characteristics of solar cells obtained by PC1D software.

\begin{tabular}{|c|c|c|c|}
\hline samples & $\mathrm{Jsc}(\mathrm{mA} / \mathrm{cm} 3)$ & $\operatorname{Voc}(\mathrm{mV})$ & $\eta(\%)$ \\
\hline $0.05 \mathrm{M}$ & 26,8 & 526,1 & 11,1 \\
\hline $0.1 \mathrm{M}$ & 28,19 & 527,8 & 11,6 \\
\hline $0.15 \mathrm{M}$ & 29,86 & 531,1 & 12,3 \\
\hline $0.2 \mathrm{M}$ & 34,13 & 536,4 & 14,3 \\
\hline 0.25 & 34,77 & 536,9 & 14,5 \\
\hline
\end{tabular}

Table 4 Photovoltaic parameters from illuminated (I-V) characteristics of obtained solar cells by experiment [10]

\begin{tabular}{|c|c|c|c|}
\hline samples & $\mathrm{Jsc}(\mathrm{mA} / \mathrm{cm} 3)$ & $\operatorname{Voc}(\mathrm{mV})$ & $\eta(\%)$ \\
\hline $0.05 \mathrm{M}$ & 23,4 & 551 & 7,8 \\
\hline $0.1 \mathrm{M}$ & 23 & 552 & 9,1 \\
\hline $0.15 \mathrm{M}$ & 24 & 552 & 10,5 \\
\hline $0.2 \mathrm{M}$ & 28 & 553 & 10,9 \\
\hline $0.25 \mathrm{M}$ & 30 & 564 & 12 \\
\hline
\end{tabular}

\section{Conclusion}

We have proposed a single hetero-junction solar cell model based on porous silicon and ZnO using modified PC1D software. ZnO has fabricated by spray pyrolysis from a solution of Zinc nitrate with different molar concentration. The influence of molar concentration full filled on the electrical and optical proprieties. We have obtained a low reflection with a high molar concentration of precursors, where the reflectivity of the device fabricated with $0.25 \mathrm{M}$ is $9 \%$. The effective carrier lifetime also increases in which the value of this device is $90 \mu \mathrm{s}$. The different parameters introduced by PC1D software, the IQE, EQE, the $\mathrm{I}-\mathrm{V}$ and the device efficiency value of this device have improved, in which the value of efficiency is $14.5 \%$ by PC1D software and $12 \%$ in the experiment.

\section{REFERENCES}

[1] Long Yang, Bao-gai Zhai , Qing-lan Ma, Yuan Ming Huang," Effect of ZnO decoration on the photovoltaic performance of TiO2 based dye sensitized solar cells," Journal of Alloys and Compounds, pp 109-112 2014. 
Transactions on Machine Learning and Artificial Intelligence Vol 5 No 4, Aug 2017

[2] Z.Yamlahi Alami,M. Salem, M. Gaidi, J.Elkhamkhami, "Effect of Zn concentration on structural and optical proprieties of $\mathrm{ZnO}$ thin films deposited by spray pyrolysis" Advanced Energy: An International Journal (AEIJ), Vol. 2, No. 4, 2015.

[3] Brijesh Kumar, Sang-Woo Kim,"Energy harvesting based on semiconducting piezoelectric ZnO nanostructures," Nano Energy ,vol 1, pp 342-355, 2012.

[4] Daqiang Gao, Zhaohui Zhang, Junli Fu, Yan Xu, Jing Qi and Desheng Xue, "Room temperature ferromagnetis of pure ZnO nanoparticles“ J. Appl. Phys, vol 105, p 113928,2009 .

[5] A.J. Behan, J.R. Neal, R.M. Ibrahim, A. Mokhtari, M. Ziese, H.J. Blythe, A.M. Fox and G.A. Gehring, "Magneto-optical and transport studies of ZnO-based dilute magnetic semiconductors," Journal of Magnetism and Magnetic Materials, Volume 310, Issue 2, p. 2158-2160,2007

[6] C. Besleaga, G.E. Stan, A.C. Galca, L. Ion, S. Antohe, "Double layer structure of ZnO thin films deposited by RF-magnetron sputtering" Applied Surface Science 258 (2012) 8819- 8824.

[7] K. Bab, C. Lazzaroni, O. Brinza, M. Nikravech,"Effect of zinc nitrate concentration on structural and optical properties of ZnO thin filmsdeposited by Spray Plasma device," Thin Solid Films , vol 558 ,pp 62-66, 2014.

[8] Zi-Neng Ng, Kah-Yoong Chan, Thanaporn Tohsophon, "Effects of annealing temperature on ZnO and AZO films prepared by sol-gel technique"Applied Surface Science, vol 258 ,pp 9604-9609 , 2012.

[9] L. Moreno, C. Sánchez-Aké, M. Bizarro, "Double-beam pulsed laser deposition for the growth "of Alincorporated ZnO thin films"Applied Surface Science 302 (2014) 46-51.

[10] M. Salem ,Z. Yamlahi Alami ,B. Bessais , A. Chahboun , M. Gaidi, "Structural and optical properties of ZnO nanoparticles deposited on porous silicon for mc-Si passivation" J Nanopart Res .17 (2015) 137

[11] B. Elidrissi, M. Addou, M. Regragui, A. Bougrine, Lebanese Sci. Res. Vol 4, p 230, 1998

[12] M.Belarbi ,A.Benyoucef ,B.Benyoucef, "Simulation of the solar cells with PC1D, application to cells based on silicon ," Advanced Energy: An International Journal (AEIJ), Vol. 1, No. 3,2014 .

[13] Babar Hussain, Abasifreke Ebong, "Specifications of ZnO growth for heterostructure solar cell and PC1D based simulations", Data in Brief volume 5, pp 516-521, 2015.

[14] Babar Hussain, Abasifreke Ebong,lan ferguson ,"zinc oxide as an active n-layer and antireflection coating for silicon based heterojunction solar cell ," solar energy materials and solar cells, vol 139 , pp 95100,2015 